\title{
miR-152 is involved in the proliferation and metastasis of ovarian cancer through repression of ERBB3
}

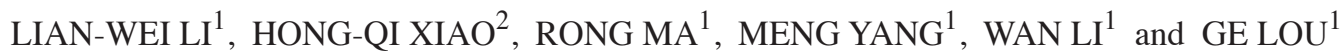 \\ ${ }^{1}$ Department of Gynecology, Harbin Medical University Cancer Hospital, Harbin, Heilongjiang 150081; \\ ${ }^{2}$ Department of General Surgery, The Fourth Affiliated Hospital of Harbin Medical University, \\ Harbin, Heilongjiang 150001, P.R. China
}

Received April 27, 2016; Accepted November 29, 2017

DOI: $10.3892 /$ ijmm.2017.3324

\begin{abstract}
MicroRNAs (miRNAs) participate in post-transcriptional regulation by targeting the 3 ' untranslated region of target genes that are involved in diverse biological processes. To the best of our knowledge, the association between miR-152 and ERBB3 in ovarian cancer remains unclear. In the present study, a negative correlation between miR-152 and ERBB3 in ovarian cancer was observed. The luciferase reporter gene assay results demonstrated that miR-152 negatively regulated ERBB3 in SKOV3 and OVCAR3 ovarian cancer cells. Furthermore, our results revealed that miR-152 suppressed the ability of ovarian cancer cell proliferation, migration and invasion, and promoted apoptosis through inhibiting ERBB3 in vitro. Therefore, in the present study, miR-152 was found to be involved in the proliferation and metastasis of ovarian cancer cells through repression of ERBB3 expression. Therefore, miR-152 may be a potential therapeutic target for the treatment of ovarian cancer.
\end{abstract}

\section{Introduction}

Ovarian cancer comprises a large proportion of all gynecological cancers, is associated with a high mortality rate, and represents a major concern for women's health worldwide (1-5). The currently applied treatments for ovarian cancer mainly include radiotherapy, chemotherapy and/or chemoradiotherapy combined with surgery. However, the lack of early symptoms and effective biomarker screening make diagnosis difficult, with $>70 \%$ of the diagnosed patients presenting with advanced epithelial ovarian carcinoma with distant metastases (6-8). At present, the molecular pathogenesis of ovarian cancer remains poorly understood. In addition, the lack of diagnostic biomarkers and effective therapeutic methods represents a major challenge in the management of this disease. Therefore, the molecular mechanism underlying the development of ovarian cancer must be urgently elucidated.

Correspondence to: Dr Ge Lou, Department of Gynecology, Harbin Medical University Cancer Hospital, 150 Haping Road, Nangang, Harbin, Heilongjiang 150081, P.R. China

E-mail: geloudoctor@sina.com

Key words: miR-152, ERBB3, ovarian cancer, migration and invasion
MicroRNAs (miRNAs), a type of endogenous, short and non-coding RNAs consisting of $\sim 20$ nucleotides, are involved in post-transcriptional regulation by targeting the 3 ' untranslated region (UTR) of target genes and affect biological processes (6,9-13). miRNAs have been found to act as a class of regulatory factors, and are closely associated with the development and progression of diverse diseases, such as cancer $(7,11,14-17)$. Various miRNAs have been reported to be involved in the development of ovarian cancer (18-25). The mechanism and function of miR-152 have been investigated in gastrointestinal (26), endometrial (27), liver (28), prostate (29) and ovarian cancer (30). However, the functional relevance of miR-152 and ERBB3 in ovarian cancer remains unknown.

The aim of the present study was to investigate whether miR-152 downregulates ERBB3 in SKOV3 and OVCAR3 ovarian cancer cells in vitro, suppressing their ability to proliferate, migrate and invade and promoting their apoptosis, in order to determine whether miR-152 is a potential therapeutic target for ovarian cancer.

\section{Materials and methods}

Clinical specimens and cell lines. The clinical samples for the present study were obtained from 36 patients with ovarian cancer in The Affiliated Tumor Hospital of Harbin Medical University (Harbin, China) between 2015 and 2016. Informed consent was obtained from all patients and the study protocol was approved by the Ethics Committee of The Affiliated Tumor Hospital of Harbin Medical University. The histological diagnosis of ovarian cancer was evaluated according to the World Health Organization. The ovarian carcinoma cell lines (SKOV3 and OVCAR3) were obtained from the American Type Culture Collection (Manassas, VA, USA).

Transfection of miR-152 mimics. Transfection of miR-152 mimics was performed as previously described (31). The primer sequence of the negative control (NC) was 5'-UUC UCCGAACGUGUCACGUTTACGUGACACGUUCGGAGA ATT-3'. miR-152-mimics with the following sequence were also designed: 5'-UCAGUGCAUGACAGAACUUGGAAG UUCUGUCAUGCACUGAUU-3'. The RNA nucleotides were synthesized by GenePharma (Shanghai, China). 
EERB3 transfection. According to the manuscript protocol, pcDNA3.1 expressing ERBB3 or control vector were transfected into SKOV3 and OVCAR3 cells that had been transfected with miR-152-NC and miR-152-mimics using the FuGENE6 (Roche Diagnostics, Mannheim, Germany) reagent. Stable transfection cell lines were screened in medium containing $1 \mathrm{mg} / \mathrm{ml} \mathrm{G} 418$ (Sigma-Aldrich; Merck KGaA, St. Louis, MO, USA).

Quantitative polymerase chain reaction (qPCR). As described previously (32), the SYBR-Green PCR Master Mix kit (Takara, Dalian, China) was used to measure the mRNA expression levels. The primer sequences for glyceraldehyde 3-phosphate dehydrogenase (GAPDH), which was used as internal control, were as follows: Forward, 5'-TGTTCGTCATGGGTGTGA AC-3' and reverse, 5'-ATGGCATGGACTGTGGTCAT-3'; the primer sequences for ERBB3 were: Forward, 5'-GCAGAT CAGTGTGTAGCGTG-3' and reverse, 5'-CGTGTGCAGTTG AAGTGACA-3'; the primer sequences for miR-152 were: Forward, 5'-TCAGTGCATGACAGAACTTGGAA-3' and reverse, 5'-GCTGTCAACGATACGCTACGT-3'; and the primer sequences for U6 were: Forward, 5'-CTCGCTTCG GCAGCACA-3' and reverse, 5'-AACGCTTCACGAATTTGC GT-3'. The Bio-Rad IQTM5 Multicolor Real-Time PCR detection System (Bio-Rad, Hercules, CA, USA) was used for qPCR. The PCR programs were set as follows: $95^{\circ} \mathrm{C}$ for $30 \mathrm{sec}$ as the first step in a loop; $95^{\circ} \mathrm{C}$ for $5 \mathrm{sec}, 60^{\circ} \mathrm{C}$ for $34 \mathrm{sec}$ as the second step, a total of 40 cycles. GAPDH or U6 were used as the internal reference, and the $2^{-\Delta \Delta \mathrm{Ct}}$ method was applied for calculating expression (1).

Western blot analysis. Whole lysates of the treated SKOV3 and OVCAR3 cells were prepared by scraping cells with RIPA buffer supplemented with a protease inhibitor cocktail (P8340; Sigma-Aldrich; Merck KGaA), then separated on $8 \%$ sodium dodecyl sulfate-polyacrylamide gel electrophoresis gels. The primary antibodies used in this study were anti-ERBB3 (1:200; Abcam, Cambridge, CA, USA) and anti-GAPDH (1:1,000; Abcam, Cambridge, UK).

Luciferase reporter assay. The binding sites between miRNAs and ERBB3 were predicted by the TargetScan database (http://www.t argetscan.org). SKOV3 and OVCAR3 cells $\left(5 \times 10^{4}\right.$ cells/well) that were co-transfected with wild-type or mutated ERBB3 and miR-152 or anti-miR-152, respectively, were incubated in 24-well plates and co-transfected with the constructed ERBB3 plasmids by Lipofectamine 2000 (using Renilla plasmid as internal reference) for $48 \mathrm{~h}$. According to the manufacturer's instructions, the luciferase activities were determined by the Dual-Luciferase Reporter assay kit (Promega, Madison, WI, USA)

MTT and colony-forming unit (CFU) assays. The MTT assay was used to determine the proliferative ability of the cells. First, the treated SKOV3 and OVCAR3 cells (3,000 cells/well) were prepared and seeded in 96-well plates with the complete media. At 0, 12, 24 and $48 \mathrm{~h}, 20 \mu \mathrm{l}$ MTT solution $(5 \mathrm{mg} / \mathrm{ml})$ were added to each well. After $4 \mathrm{~h}$ of incubation, the supernatant was discarded, and $100 \mu 1$ dimethyl sulfoxide solution was added to dissolve the crystals. A microplate reader (BioTek Instruments, Inc., Winooski, VT, USA) was used to detect the absorbance at $570 \mathrm{~nm}$. The treated SKOV3 and OVCAR3 cells were incubated for 10 days. Methanol was used to fix the forming colonies and Giemsa was used to dye the colonies. The CFUs in the treatment and the control groups were counted.

Flow cytometric analysis of cell apoptosis. The Annexin V-FITC Apoptosis Detection kit II (BD Pharmingen, San Diego, CA, USA) was used to detect apoptotic cells according to the manufacturer's protocol. Briefly, the treated SKOV3 and OVCAR3 cells were resuspended with binding buffer at a density of $1 \times 10^{6} \mathrm{cells} / \mathrm{ml}$. Annexin V-FITC (5 $\left.\mu \mathrm{l}\right)$ and propidium iodide $(1 \mu \mathrm{g} / \mathrm{ml})$ were used to stain the cells. The flow cytometry results were analyzed by the FlowJo software (Tree Star Corp., Ashland, OR, USA). Cells were grouped into the viable, early-stage apoptotic, late-stage apoptotic and dead cell groups.

Wound healing assay. As described previously (33), the treated SKOV3 and OVCAR3 cells were incubated in 6-well plates. Small wounds were formed by scratching a line of cells. The small fragments of cells were gently washed with phosphate-buffered saline. After 24 and $72 \mathrm{~h}$, the wounds were photographed under a microscope. The distance of cells from the edge of the scratch represented the degree of cell migration.

Migration and invasion assays. According to the manufacturer's instructions, the treated SKOV3 and OVCAR3 cells were starved overnight in serum-free medium and resuspended at a density of $2.5 \times 10^{6} \mathrm{cells} / \mathrm{ml}$ in serum-free medium. Treated cells $(200 \mu \mathrm{l})$ were seeded into the top chamber of the $8-\mu \mathrm{m}$ pore cell culture insert, and complete medium was added into the bottom chamber. After $24 \mathrm{~h}$, the upper chamber cells were removed using a cotton swab and the migrating cells were fixed with $4 \%$ paraformaldehyde and stained with $0.1 \%$ crystal violet solution. The migrating cells were counted at 5 random fields. For the invasion assay, diluted Matrigel (BD Biosciences, San Diego, CA, USA) was paved into the upper well of the Transwell chamber (Corning, Inc., Corning, NY, USA) and incubated for $2 \mathrm{~h}$ at $37^{\circ} \mathrm{C}$.

Tumor formation in nude mice. This study was approved by the Institutional Committee for Animal Research and completed in accordance with the Institutional Animal Care and Use Committee. Treated SKOV3 cells (100 $\mu 12 \times 10^{7} /$ mouse) were subcutaneously injected into the flanks of 5-week-old BALB/c male athymic nude mice for a total of 5 times over 4 weeks. At the specified time, the treated nude mice were sacrificed and the tumors were collected and measured.

Statistical analysis. All data are presented as the mean \pm standard deviation from three independent experiments. $\mathrm{P}<0.05$ indicates statistically significant differences. Statistical significance was analyzed using GraphPad (GraphPad Prism Software, La Jolla, CA, USA) and the SPSS 20.0 software (variance and Student's t-test).

\section{Results}

Screening of miRNAs that are closely associated with ERBB3 in ovarian cancer. In order to study the regulatory mechanism of the ERBB3 gene in ovarian cancer, the miRNA target sites in ERBB3 3'UTR were predicted by TargetScan. We found multiple miRNAs displaying target sites in ERBB3 
A Human ERBB3 3 ' UTR

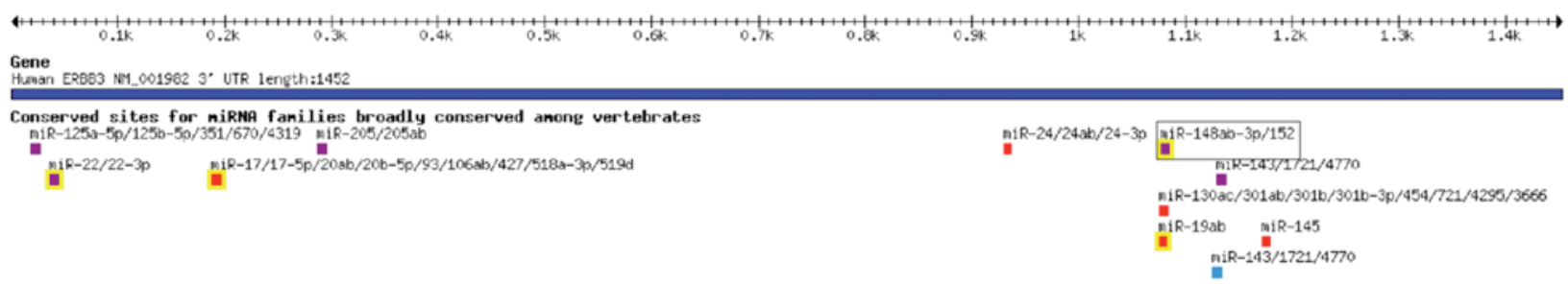
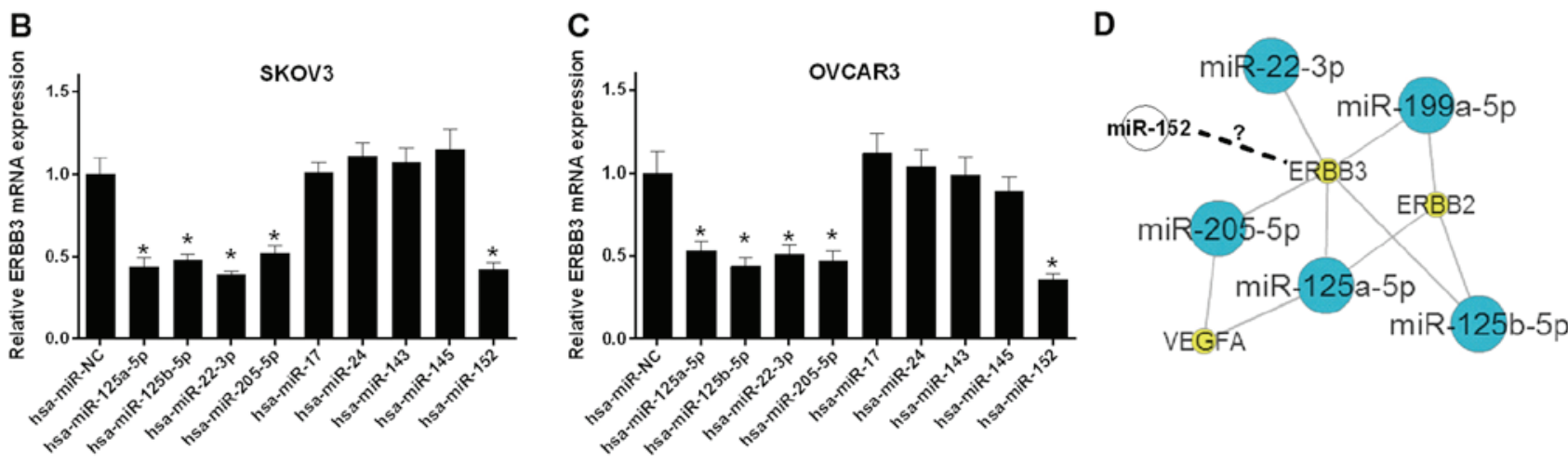

Figure 1. miRNAs that are closely associated with ERBB3 were screened in ovarian cancer. (A) Diagrams of predicted miRNA target sites in ERBB3 3'UTR (TargetScan). (B) The mRNA expression levels of ERBB3 were determined by reverse transcription-quantitative polymerase chain reaction (RT-qPCR) in SKOV3 cells. U6 was used as a reference mRNA. (C) The mRNA expression levels of ERBB3 3'UTR were measured by RT-qPCR in OVCAR3 cells. U6 was used as a reference mRNA. (D) Diagram of the interactions between ERBB3 and relevant miRNAs. UTR, untranslated region.
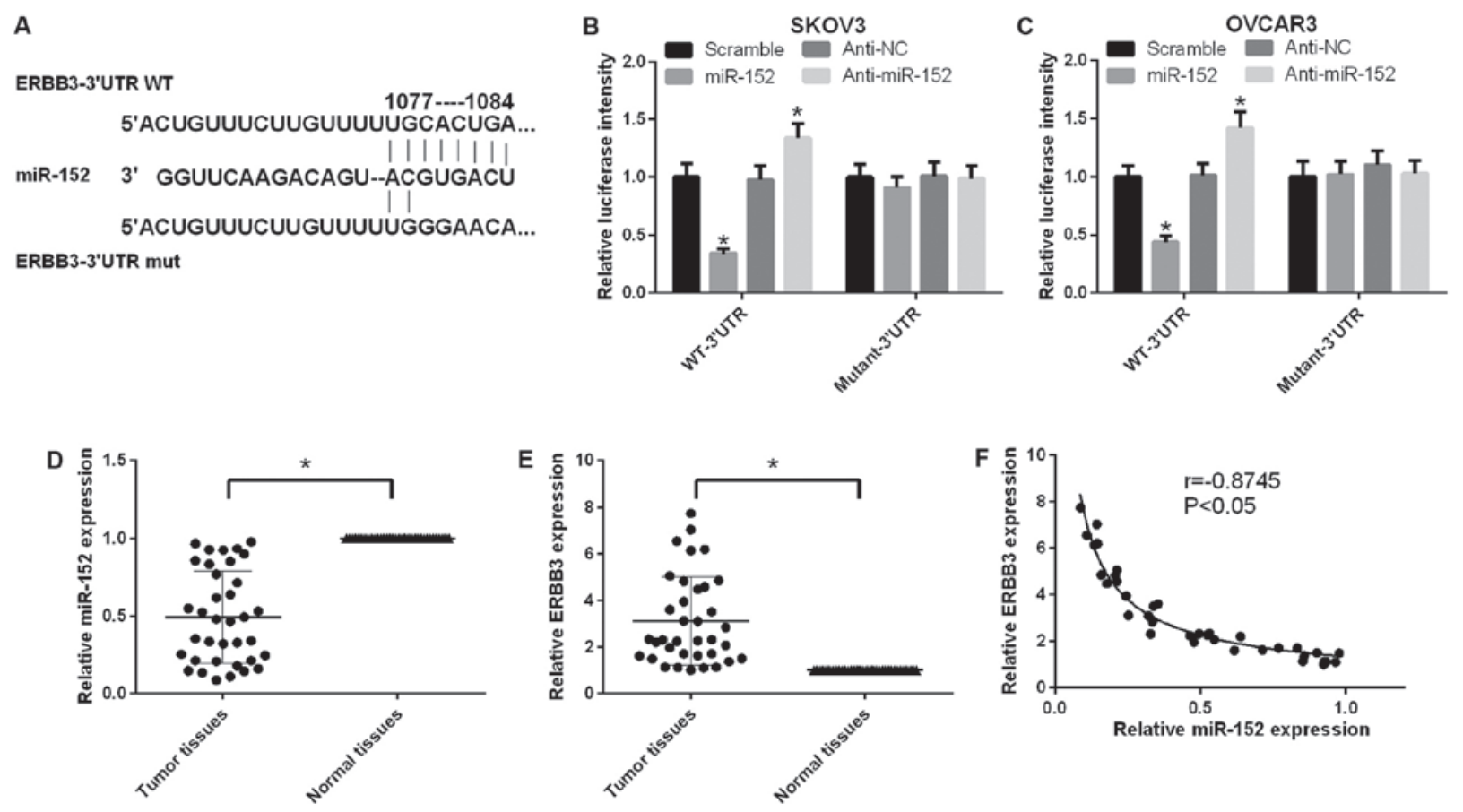

Figure 2. miR-152 downregulates ERBB3 expression in human ovarian cancer. (A) As predicted by TargetScan, the miR-152 target site in the sequence of ERBB3 and the mutational site of ERBB3 are shown. (B) The luciferase reporter gene assay was used to measure the activity of ERBB3 in SKOV3 cells that were co-transfected with wild-type or mutated ERBB3 and miR-152 or anti-miR-152, respectively $(\mathrm{P}<0.05)$. (C) The luciferase reporter gene assay was used to measure the activity of ERBB3 in OVCAR3 cells that were cotransfected with wild-type or mutated ERBB3 and miR-152 or anti-miR-152, respectively $(\mathrm{P}<0.05)$. (D) Reverse transcription-quantitative polymerase chain reaction (RT-qPCR) was used to determine the expression level of miR-152 in ovarian cancer and adjacent ovarian tissues $(\mathrm{P}<0.05)$. (E) The expression level of ERBB3 was measured by RT-qPCR in ovarian cancer and adjacent ovarian tissues $(\mathrm{P}<0.05)$. (F) Scatter diagram indicating the correlation between ERBB3 and miR-152 in ovarian cancer and adjacent ovarian tissues by RT-qPCR $(r=-0.8745, \mathrm{P}<0.05)$.

3'UTR (Fig. 1A). Furthermore, the mRNA expression levels of ERBB3 were detected in SKOV3 cells transfected with miRNAs. The results revealed that the miR-125a-5p, miR-125b-5p, miR-22-3p, miR-205-5p and miR-152-trans- 

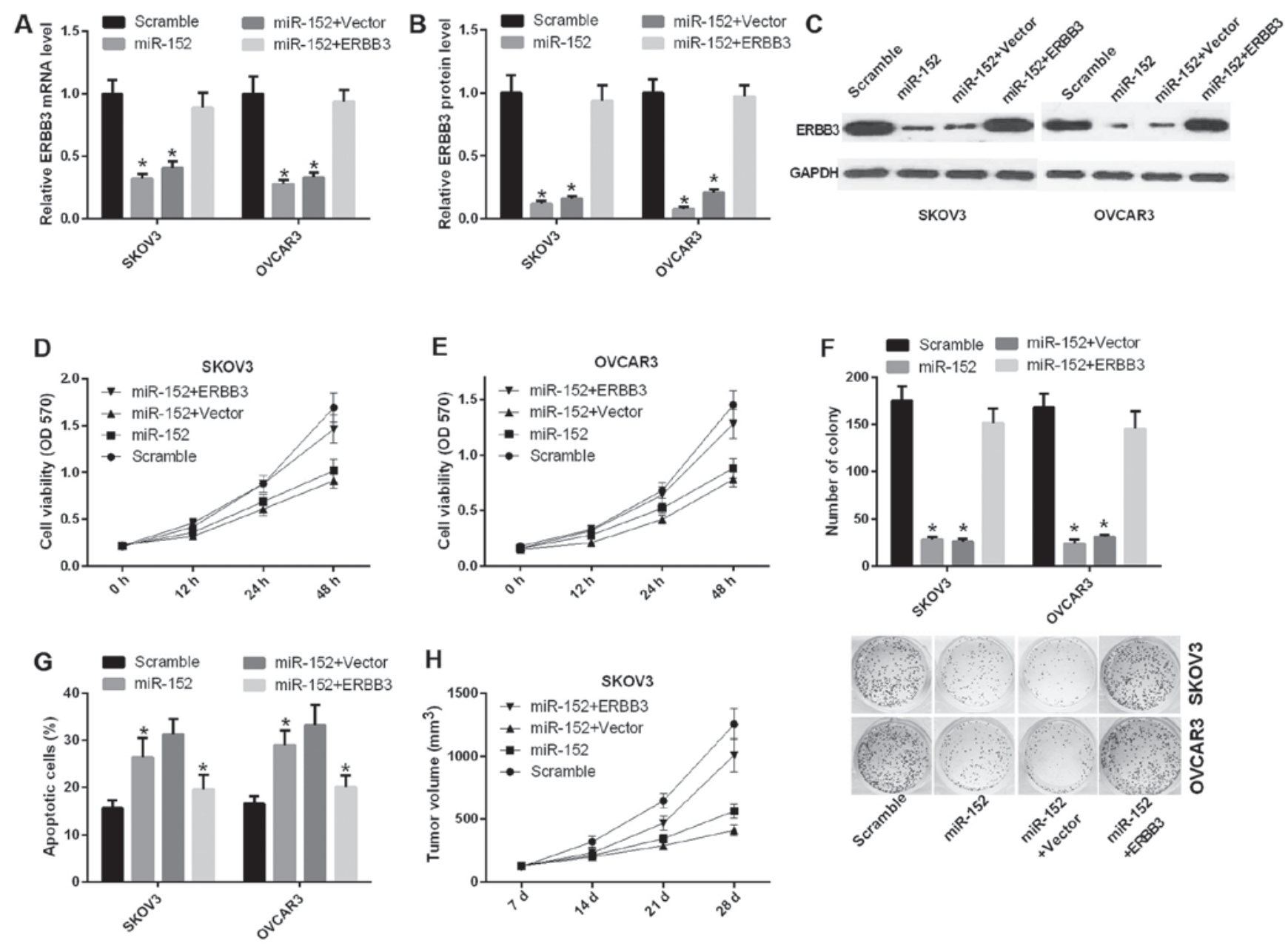

Figure 3. miR-152 suppresses the ability of ovarian cancer proliferation and promotes apoptosis through inhibiting ERBB3. (A) Reverse transcription-quantitative polymerase chain reaction was used to determine the mRNA expression level of ERBB3 in SKOV3 and OVCAR3 cells transfected with scramble, miR-152, miR-152 and vector, or miR-152 and ERBB3 ( $\mathrm{P}<0.05$ ). (B and C) Analysis of the ERBB3 protein expression level in SKOV3 and OVCAR3 cells $(\mathrm{P}<0.05)$. (D and E) The MTT assay was performed to determine the proliferation capacity of SKOV3 and OVCAR3 cells transfected with scramble, miR-152, miR-152 and vector, or miR-152 and ERBB3. (F) The capacity of cell proliferation was analyzed by colony formation evaluation in SKOV3 and OVCAR3 cells $(\mathrm{P}<0.05)$. (G) The number of apoptotic cells was counted in SKOV3 and OVCAR3 cells transfected with scramble, miR-152, miR-152 and vector, or miR-152 and ERBB3 $(\mathrm{P}<0.05)$. $(\mathrm{H})$ Athymic mice were treated with SKOV3 cells transfected with scramble, miR-152, miR-152 and vector, or miR-152 and ERBB3 for 7, 14, 21 and 28 days. The tumor volume was measured and calculated at the specified timepoints. OD, optical density.

fected SKOV3 cells have a significantly lower level of ERBB3 mRNA ( $\mathrm{P}<0.05$; Fig. 1B). A similar tendency was observed in OVCAR3 cells $(\mathrm{P}<0.05$; Fig. 1C). ERBB3 interacted with miR-125a-5p, miR-125b-5p, miR-22-3p and miR-205-5p in the oncomiRDB datebase (http://bioinfo.au.tsinghua.edu. $\mathrm{cn} / \mathrm{member} /$ jgu/oncomirdb/index.php) (Fig. 1D). However, the association between miR-152 and ERBB3 remains unclear.

miR-152 downregulates ERBB3 expression in human ovarian cancer. According to the TargetScan prediction, wild-type and mutated ERBB3 vectors were structured and cotransfected with miR-152 or anti-miR-152 in SKOV3 cells, respectively. The miR-152 target site in the sequence of ERBB3 and the mutational site of ERBB3 are shown in Fig. 2A. It was indicated that miR-152 negatively regulated ERBB3 using the luciferase reporter gene assay in SKOV3 cells $(\mathrm{P}<0.05$; Fig. $2 \mathrm{~B})$. A similar tendency was observed in OVCAR3 cells (Fig. 2C). In addition, the expression level of miR-152 in ovarian cancer tissues was found to be lower compared with that in adjacent non-cancerous tissues (P<0.05; Fig. 2D). Furthermore, the expression level of
ERBB3 in ovarian cancer tissues was higher compared with that in adjacent non-cancerous tissues $(\mathrm{P}<0.05 ;$ Fig. $2 \mathrm{E})$. Further analysis confirmed a negative correlation between miR-152 and ERBB3 in ovarian cancer tissues $(\mathrm{P}<0.05, \mathrm{r}=0.8745$; Fig. $2 \mathrm{~F})$.

miR-152 suppresses the ability of ovarian cancer cell proliferation and promotes apoptosis through inhibiting ERBB3. To further study the function of miR-152 in ovarian cancer, functional assays were performed in ovarian cancer cell lines. The mRNA expression level of ERBB3 was decreased in SKOV3 and OVCAR3 cells transfected with miR-152 compared with scramble, and was significantly increased in SKOV3 and OVCAR3 cells transfected with both miR-152 and ERBB3 relative to the control group $(\mathrm{P}<0.05)$ (Fig. 3A). Similarly, the protein level of ERBB3 was also reduced by ERBB3 vector in SKOV3 and OVCAR3 cells (Fig. 3B and C). Furthermore, the cell proliferation ability was decreased in SKOV3 cells transfected with miR-152 compared with scramble, and this ability was significantly increased in SKOV3 cells transfected with both miR-152 and ERBB3 relative to both miR-152 and vector (Fig. 3D). 

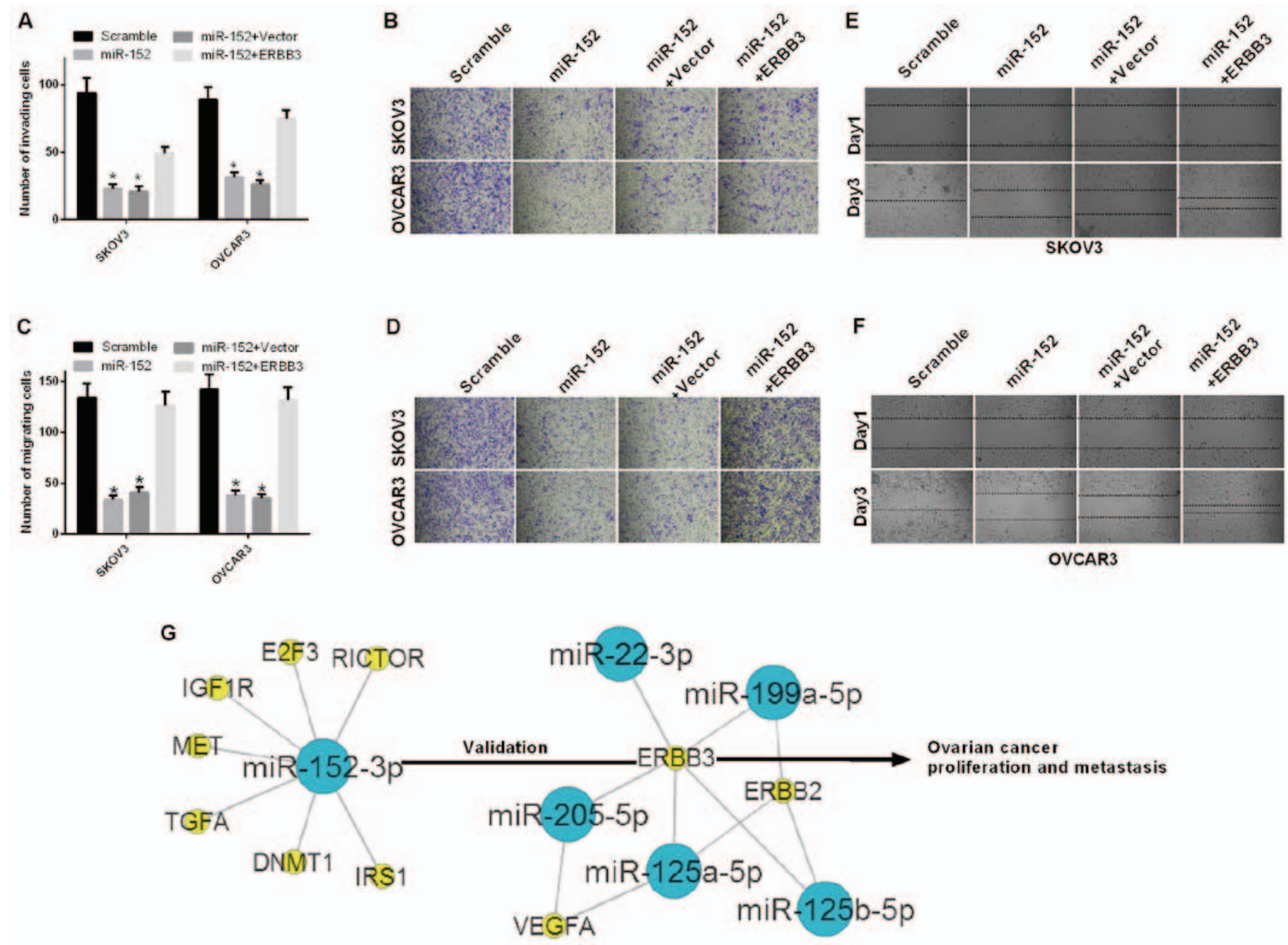

Figure 4. miR-152 inhibits the ability of migration and invasion of ovarian cancer cells through ERBB3. (A and B) The number of invading cells was counted among SKOV3 and OVCAR3 cells transfected with scramble, miR-152, miR-152 and vector, or miR-152 and ERBB3 (P<0.05). (C and D) The migration assay was performed in SKOV3 and OVCAR3 cells transfected with scramble, miR-152, miR-152 and vector, or miR-152 and ERBB3. Magnification, $\mathrm{x} 200$ ( $\mathrm{P}<0.05)$. (E and F) The migration ability of SKOV3 and OVCAR3 cells that were transfected with scramble, miR-152, miR-152 and vector, or miR-152 and ERBB3 was measured by the wound healing assay at 1 and 3 days. $(G)$ The gene network between miR-152 and ERBB3 was established in ovarian cancer. Blue nodes, miRs; yellow nodes, target genes.

A similar result was observed in OVCAR3 cells (Fig. 3E). Similarly, the CFU assay results indicated that miR-152 inhibited the proliferation ability of SKOV3 and OVCAR3 cells through ERBB3 (Fig. 3F). Apoptosis assay proved that miR-152 accelerated the apoptosis of SKOV3 and OVCAR3 cells, and that ERBB3 can reverse miR-152-mediated promotion of apoptosis in vitro (Fig. 3G). Furthermore, to detect the effect of miR-152 on tumorigenesis in vivo, SKOV3 cells transfected with scramble, miR-152, miR-152 and vector, or miR-152 and ERBB3 were implanted subcutaneously into nude mice. The results indicated that miR-152 markedly inhibited tumor growth, while ERBB3 was able to reverse this phenotype (Fig. $3 \mathrm{H}$ ).

miR-152 inhibits the ability of migration and invasion in ovarian cancer cells through ERBB3. The effect of miR-152 on cell migration and invasion ability of ovarian cancer cells was further investigated. First, SKOV3 and OVCAR3 cells were transfected with scramble, miR-152, miR-152 and vector, or miR-152 and ERBB3. The results of the invasion assay demonstrated that the invasion ability was significantly decreased in SKOV3 and OVCAR3 cells transfected with
miR-152 compared with scramble, and significantly increased in SKOV3 cells transfected with both miR-152 and ERBB3 relative to both miR-152 and vector ( $\mathrm{P}<0.05$; Fig. $4 \mathrm{~A}$ and $\mathrm{B})$. The results of the migration assay also demonstrated that the migration ability was significantly decreased in SKOV3 and OVCAR3 cells transfected with miR-152 compared with scramble, and significantly increased in SKOV3 cells transfected with both miR-152 and ERBB3 relative to both miR-152 and vector $(\mathrm{P}<0.05$; Fig. $4 \mathrm{C}$ and $\mathrm{D})$. In addition, the migration ability of SKOV3 and OVCAR3 cells transfected with scramble, miR-152, miR-152 and vector, or miR-152 and ERBB3, were evaluated by the wound healing assay at 1 and 3 days. The results indicated that miR-152 inhibited the migration ability of SKOV3 and OVCAR3 cells through ERBB3 (Fig. 4E and F).

Regulatory mechanism underlying the interaction between miR-152 and ERBB3 in ovarian cancer. The gene network between miR-152 and ERBB3 was established in ovarian cancer as shown in Fig. 4G. miR-152 downregulated the expression level of ERBB3 by targeting the ERBB3 promoter-binding site 
and inhibited ovarian cancer cell proliferation, migration and invasion, while promoting apoptosis in vitro.

\section{Discussion}

Accumulating evidence reported that miRNAs play key roles in human cancer, including cancer cell proliferation, metastasis, inflammation and angiogenesis $(13,34)$. Recent studies proved that miR-152 served as a tumor inhibitor, and could be silenced by DNA hypermethylation in endometrial cancer (27). The combined effect of miR-152 and miR-185 played an important role in the treatment of ovarian cancer by targeting DNMT1, independent of decitabine (35). miR-152 affected the proliferation ability of ovarian cancer cells (30), whereas miR-152 reduced the invasion and angiogenesis capacity of glioma cells (36). Downregulated miR-152 affected aberrant DNA methylation in hepatitis B virus-related hepatocellular carcinoma (28). miR-152 inhibited migration and invasion through TGF- $\alpha$ in prostate cancer cells (29). However, their mechanism of action is not entirely clear in ovarian cancer. In the present study, miR-152 expression was found to be lower in ovarian cancer tissues compared with that in adjacent non-cancerous tissues. miR-152 suppressed the ability of ovarian cancer proliferation, migration and invasion, and promoted apoptosis in vitro.

ERBB3 is one of the four members of ERBB family, which may transduce extracellular signals into the cell, resulting in several changes in the regulatory processes of various cancer cells, including proliferation, survival, apoptosis, migration and invasion (37-39). It has been demonstrated that ERBB3 significantly downregulates kinase activity (40). ERBB3 overexpression has been proven to take part in the regulation of various physiological and pathological pathways in several types of cancer, such as melanoma, breast, prostate, lung and pancreatic cancer (41-44). It has also been reported that ERBB3 may activate downstream phosphatidylinositol-3-kinase, which is associated with tumorigenesis and resistance to therapy (45). Therefore, an increasing number of studies indicate that ERBB3 is a potential target of cancer therapy. In the present study, ERBB3 expression was found to be higher in ovarian cancer tissues compared with that in adjacent non-cancerous tissues. There was a negative correlation between miR-152 and ERBB3 expression in ovarian cancer tissues. ERBB3 was downregulated by miR-152 in ovarian cancer cells. ERBB3 may reverse miR-152 mediated-inhibition of ovarian cancer proliferation, migration and invasion, and miR-152-mediated promotion of apoptosis in vitro. ERBB3 may also reverse miR-152-mediated inhibition of tumor growth in vivo.

In conclusion, the findings of the present study indicated that miR-152 is involved in the regulation of the proliferation and metastasis of ovarian cancer cells through repression of ERBB3 expression. First, it was demonstrated that the mRNA expression levels of miR-125a-5p, miR-125b-5p, miR-22-3p, miR-205-5p and miR-152 were significantly downregulated in SKOV3 cells. Second, a negative correlation between miR-152 and ERBB3 was identified in ovarian cancer tissues, and miR-152 downregulated ERBB3 expression in ovarian cancer cells. Furthermore, our results demonstrated that miR-152 suppressed the ability of ovarian cancer cells to proliferate, migrate and invade, and promoted their apoptosis through inhibiting ERBB3 in vitro. Therefore, the present study demonstrated that miR-152 may be a potential therapeutic target for the treatment of ovarian cancer.

\section{Acknowledgements}

The present study was supported by the National Natural Science Foundation of China (grant no. 81472028), the Scientific Research Project of Hei Long-jiang Provincial Health Bureau of China [AN1] (no. 2016-105) and the Administration of Traditional Chinese Medicine of Heilongjiang Province, China (no. ZHY16-110).

\section{References}

1. Siegel R, Naishadham D and Jemal A: Cancer statistics, 2012. CA Cancer J Clin 62: 10-29, 2012.

2. Kim K, Zang R, Choi SC, Ryu SY and Kim JW: Current status of gynecological cancer in China. J Gynecol Oncol 20: 72-76, 2009.

3. Legge F, Ferrandina G, Salutari V and Scambia G: Biological characterization of ovarian cancer: prognostic and therapeutic implications. Ann Oncol 16 (Suppl 4): iv95-101, 2005.

4. Siegel R, Ward E, Brawley O and Jemal A: Cancer statistics, 2011: The impact of eliminating socioeconomic and racial disparities on premature cancer deaths. CA Cancer J Clin 61: 212-236, 2011.

5. Seidman JD, Soslow RA, Vang R, Berman JJ, Stoler MH, Sherman ME, Oliva E, Kajdacsy-Balla A, Berman DM and Copeland LJ: Borderline ovarian tumors: Diverse contemporary viewpoints on terminology and diagnostic criteria with illustrative images. Hum Pathol 35: 918-933, 2004.

6. Iorio MV, Visone R, Di Leva G, Donati V, Petrocca F, Casalini P, Taccioli C, Volinia S, Liu CG, Alder H, et al: MicroRNA signatures in human ovarian cancer. Cancer Res 67: 8699-8707, 2007.

7. Zaman MS, Maher DM, Khan S, Jaggi M and Chauhan SC: Current status and implications of microRNAs in ovarian cancer diagnosis and therapy. J Ovarian Res 5: 44, 2012.

8. Heintz AP, Odicino F, Maisonneuve P, Quinn MA, Benedet JL, Creasman WT, Ngan HY, Pecorelli S and Beller U: Carcinoma of the ovary. Int J Gynaecol Obstet 95 (Suppl 1): S161-S192, 2006.

9. Bartel DP: MicroRNAs: genomics, biogenesis, mechanism, and function. Cell 116: 281-297, 2004.

10. Iorio MV and Croce CM: MicroRNAs in cancer: Small molecules with a huge impact. J Clin Oncol 27: 5848-5856, 2009.

11. Farazi TA, Hoell JI, Morozov P and Tuschl T: MicroRNAs in human cancer. Adv Exp Med Biol 774: 1-20, 2013.

12. Djuranovic S, Nahvi A and Green R: A parsimonious model for gene regulation by miRNAs. Science 331: 550-553, 2011.

13. Kasinski AL and Slack FJ: Epigenetics and genetics. MicroRNAs en route to the clinic: Progress in validating and targeting microRNAs for cancer therapy. Nat Rev Cancer 11: 849-864, 2011.

14. Croce CM and Calin GA: miRNAs, cancer, and stem cell division. Cell 122: 6-7, 2005.

15. Cheng AM, Byrom MW, Shelton J and Ford LP: Antisense inhibition of human miRNAs and indications for an involvement of miRNA in cell growth and apoptosis. Nucleic Acids Res 33: 1290-1297, 2005.

16. Baer C, Claus R and Plass C: Genome-wide epigenetic regulation of miRNAs in cancer. Cancer Res 73: 473-477, 2013.

17. Di Leva G and Croce CM: The role of microRNAs in the tumorigenesis of ovarian cancer. Front Oncol 3: 153, 2013.

18. Dahiya N and Morin PJ: MicroRNAs in ovarian carcinomas. Endocr Relat Cancer 17: F77-F89, 2010.

19. Mateescu B, Batista L, Cardon M, Gruosso T, de Feraudy Y, Mariani O, Nicolas A, Meyniel JP, Cottu P, Sastre-Garau X, et al: miR-141 and miR-200a act on ovarian tumorigenesis by controlling oxidative stress response. Nat Med 17: 1627-1635, 2011.

20. Dahiya N, Sherman-Baust CA, Wang TL, Davidson B, Shih IeM, Zhang Y, Wood W III, Becker KG and Morin PJ: MicroRNA expression and identification of putative miRNA targets in ovarian cancer. PLoS One 3: e2436, 2008.

21. Hu X, Macdonald DM, Huettner PC, Feng Z, El Naqa IM, Schwarz JK, Mutch DG, Grigsby PW, Powell SN and Wang X: A miR-200 microRNA cluster as prognostic marker in advanced ovarian cancer. Gynecol Oncol 114: 457-464, 2009. 
22. Cheng W, Liu T, Wan X, Gao Y and Wang H: MicroRNA-199a targets CD44 to suppress the tumorigenicity and multidrug resistance of ovarian cancer-initiating cells. FEBS J 279: 2047-2059, 2012.

23. Laios A, O'Toole S, Flavin R, Martin C, Kelly L, Ring M, Finn SP, Barrett C, Loda M, Gleeson N, et al: Potential role of miR-9 and miR-223 in recurrent ovarian cancer. Mol Cancer 7: 35, 2008.

24. Nagaraja AK, Creighton CJ, Yu Z, Zhu H, Gunaratne PH, Reid JG, Olokpa E, Itamochi H, Ueno NT, Hawkins SM, et al: A link between mir-100 and FRAP1/mTOR in clear cell ovarian cancer. Mol Endocrinol 24: 447-463, 2010.

25. Fu X, Tian J, Zhang L, Chen Y and Hao Q: Involvement of microRNA-93, a new regulator of PTEN/Akt signaling pathway, in regulation of chemotherapeutic drug cisplatin chemosensitivity in ovarian cancer cells. FEBS Lett 586: 1279-1286, 2012.

26. Chen Y, Song Y, Wang Z, Yue Z, Xu H, Xing C and Liu Z: Altered expression of MiR-148a and MiR-152 in gastrointestinal cancers and its clinical significance. J Gastrointest Surg 14: 1170-1179, 2010.

27. Tsuruta T, Kozaki K, Uesugi A, Furuta M, Hirasawa A, Imoto I, Susumu N, Aoki D and Inazawa J: miR-152 is a tumor suppressor microRNA that is silenced by DNA hypermethylation in endometrial cancer. Cancer Res 71: 6450-6462, 2011.

28. Huang J, Wang Y, Guo Y and Sun S: Down-regulated microRNA-152 induces aberrant DNA methylation in hepatitis B virus-related hepatocellular carcinoma by targeting DNA methyltransferase 1. Hepatology 52: 60-70, 2010.

29. Zhu C, Li J, Ding Q, Cheng G, Zhou H, Tao L, Cai H, Li P, Cao Q, $\mathrm{Ju} \mathrm{X}$, et al: miR-152 controls migration and invasive potential by targeting TGF $\alpha$ in prostate cancer cell lines. Prostate 73 1082-1089, 2013

30. Zhou X, Zhao F, Wang Z-N, Song YX, Chang H, Chiang Y and Xu HM: Altered expression of miR-152 and miR-148a in ovarian cancer is related to cell proliferation. Oncol Rep 27: 447-454, 2012.

31. Lim LP, Lau NC, Garrett-Engele P, Grimson A, Schelter JM, Castle J, Bartel DP, Linsley PS and Johnson JM: Microarray analysis shows that some microRNAs downregulate large numbers of target mRNAs. Nature 433: 769-773, 2005.

32. Jiang L, Lai YK, Zhang J, Wang H, Lin MC, He ML and Kung HF: Targeting S100P inhibits colon cancer growth and metastasis by Lentivirus-mediated RNA interference and proteomic analysis. Mol Med 17: 709-716, 2011

33. Madhyastha HK, Radha KS, Nakajima Y, Omura $S$ and Maruyama M: uPA dependent and independent mechanisms of wound healing by C-phycocyanin. J Cell Mol Med 12: 2691-2703, 2008.
34. Wang J, Paris PL, Chen J, Ngo V, Yao H, Frazier ML, Killary AM, Liu CG, Liang H, Mathy C, et al: Next generation sequencing of pancreatic cyst fluid microRNAs from low grade-benign and high grade-invasive lesions. Cancer Lett 356: 404-409, 2015.

35. Xiang Y, Ma N, Wang D, Zhang Y, Zhou J, Wu G, Zhao R, Huang H, Wang X, Qiao Y, et al: MiR-152 and miR-185 co-contribute to ovarian cancer cells cisplatin sensitivity by targeting DNMT1 directly: A novel epigenetic therapy independent of decitabine. Oncogene 33: 378-386, 2014.

36. Zheng X, Chopp M, Lu Y, Buller B and Jiang F: MiR-15b and miR-152 reduce glioma cell invasion and angiogenesis via NRP-2 and MMP-3. Cancer Lett 329: 146-154, 2013.

37. Sharma SV and Settleman J: ErbBs in lung cancer. Exp Cell Res 315: 557-571, 2009.

38. Hynes NE and Lane HA: ERBB receptors and cancer: The complexity of targeted inhibitors. Nat Rev Cancer 5: 341-354, 2005.

39. Hynes NE and MacDonald G: ErbB receptors and signaling pathways in cancer. Curr Opin Cell Biol 21: 177-184, 2009.

40. Shi F, Telesco SE, Liu Y, Radhakrishnan R and Lemmon MA: ErbB3/HER3 intracellular domain is competent to bind ATP and catalyze autophosphorylation. Proc Natl Acad Sci USA 107: 7692-7697, 2010

41. Ocana A, Vera-Badillo F, Seruga B, Templeton A, Pandiella A and Amir E: HER3 overexpression and survival in solid tumors: A meta-analysis. J Natl Cancer Inst 105: 266-273, 2013.

42. Cook RS, Garrett JT, Sánchez V, Stanford JC, Young C, Chakrabarty A, Rinehart C, Zhang Y, Wu Y, Greenberger L, et al: ErbB3 ablation impairs PI3K/Akt-dependent mammary tumorigenesis. Cancer Res 71: 3941-3951, 2011.

43. Wimmer E, Kraehn-Senftleben G and Issing WJ: HER3 expression in cutaneous tumors. Anticancer Res 28: 973-979, 2008.

44. Reschke M, Mihic-Probst D, van der Horst EH, Knyazev P, Wild PJ, Hutterer M, Meyer S, Dummer R, Moch H and Ullrich A: HER3 is a determinant for poor prognosis in melanoma. Clin Cancer Res 14: 5188-5197, 2008.

45. Prigent SA and Gullick WJ: Identification of c-erbB-3 binding sites for phosphatidylinositol 3'-kinase and SHC using an EGF receptor/c-erbB-3 chimera. EMBO J 13: 2831-2841, 1994.

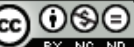

This work is licensed under a Creative Commons Attribution-NonCommercial-NoDerivatives 4.0 International (CC BY-NC-ND 4.0) License. 